\title{
Reduction of Nasal Bone Fracture using Ultrasound Imaging during Surgery
}

\author{
So-Min Hwang, \\ Hao-Ching Pan, \\ Hong-ll Kim, \\ Hyung-Do Kim, \\ Min-Kyu Hwang, \\ Min-Wook Kim, \\ Jong-Seo Lee \\ Aesthetic, Plastic and Reconstructive \\ Surgery Center, Good Moonhwa Hospital, \\ Busan, Korea
}

No potential conflict of interest relevant to this article was reported.

\begin{abstract}
Background: Most nasal bone fractures are corrected using non-invasive methods. Often, patients are dissatisfied with surgical outcomes following such closed approach. In this study, we compare surgical outcomes following blind closed reduction to that of ultrasound-guided reduction.

Methods: A single-institutional prospective study was performed for all nasal fracture patients $(n=28)$ presenting between May 2013 and November 2013. Upon research consent, patients were randomly assigned to either the control group $(n=14$, blind reduction) or the experimental group ( $n=14$, ultrasound-guided reduction). Surgical outcomes were evaluated using preoperative and 3-month postoperative $\mathrm{X}$-ray images by two independent surgeons. Patient satisfaction was evaluated using a questionnaire survey. Results: The experimental group consisted of 4 patients with Plane I fracture and 10 patients with Plane II fracture. The control group consisted of 3 patients with Plane I fracture and 11 patients with Plane II fracture. The mean surgical outcomes score and the mean patient dissatisfaction score were found not to differ between the experimental and the control group in Plane I fracture ( $p=0.755,0.578$, respectively). In a subgroup analysis consisting of Plane II fractures only, surgeons graded outcomes for ultrasoundguided reduction higher than that for the control group $(p=0.007)$. Likewise, among the Plane II fracture patients, those who underwent ultrasound-guided reduction were less dissatisfied than those who underwent blind reduction ( $p=0.043$ ).

Conclusion: Our study result suggests that ultrasound-guided closed reduction is superior to blind closed reduction in those patients with Plane II nasal fractures.
\end{abstract}

Keywords: Nasal bone / Ultrasonography / Nose

\section{INTRODUCTION}

Facial fractures are common presentations in the field of plastic surgery. Among these, nasal bone fractures occur most frequently and thus represent a significant portion of the plastic surgery practice $[1,2]$.

Management of nasal bone fractures can be largely divided

\footnotetext{
Correspondence: So-Min Hwang

Aesthetic, Plastic and Reconstructive Surgery Center, Good Moonhwa Hospital, 119 Beomil-ro, Dong-gu, Busan 48735, Korea

E-mail: lexusair@gmail.com

Received August 14, 2015 / Revised September 17, 2015 / Accepted December 3, 2015
}

into open and closed reductions. Of the two, closed reduction is the treatment of choice in most cases because of the relatively short operation time, minimal scars, tissue damage, and shorter recovery time. However, the closed approach does not allow direct visualization of the fractured site and can lead to incomplete or failed reduction. Postoperative outcomes are difficult to predict for this reason $[1,3,4]$.

Reductions can be visualized intraoperatively using portable X-ray, ultrasound, and C-arm fluoroscopy. Among these methods, we investigated whether the use of intraoperative ultrasound is associated with improved outcomes following closed nasal bone reduction $[1,3-5]$. 


\section{METHODS}

A single-institutional prospective study was performed for nasal fracture patients $(\mathrm{n}=28)$ presenting between May 2013 and November 2013. Upon research consent, patients were randomly assigned to either the control group (blind reduction, $\mathrm{n}=14$ ) or the experimental group (ultrasound guided reduction, $n=14$ ). Patients were excluded for Plane III fractures in the Stranc classification, as these patient would require open reduction.

Reduction operation was usually performed 7 days after trauma, under general anesthesia. For the control group, the closed reduction was performed after evaluating the fracture with palpation and the necessity of reduction using the conventional manner, that is, only by the surgeon's sense of touch. For the experimental group, the nasal bone was reduced while visualizing the fracture in longitudinal and transverse views (Fig. 1) using a 2D ultrasound probe (Accuvix V10, 8.0-MHz linear probe, L513IS, Samsung, Seoul, Korea). All operations were performed by a single surgeon.

After each reduction, the nasal cavities were packed with Merocel (Medtronic, Minnesota, USA) for 5 days. Patients were asked to use a Denver nasal splint (JMEDICS, Cundinamarca,
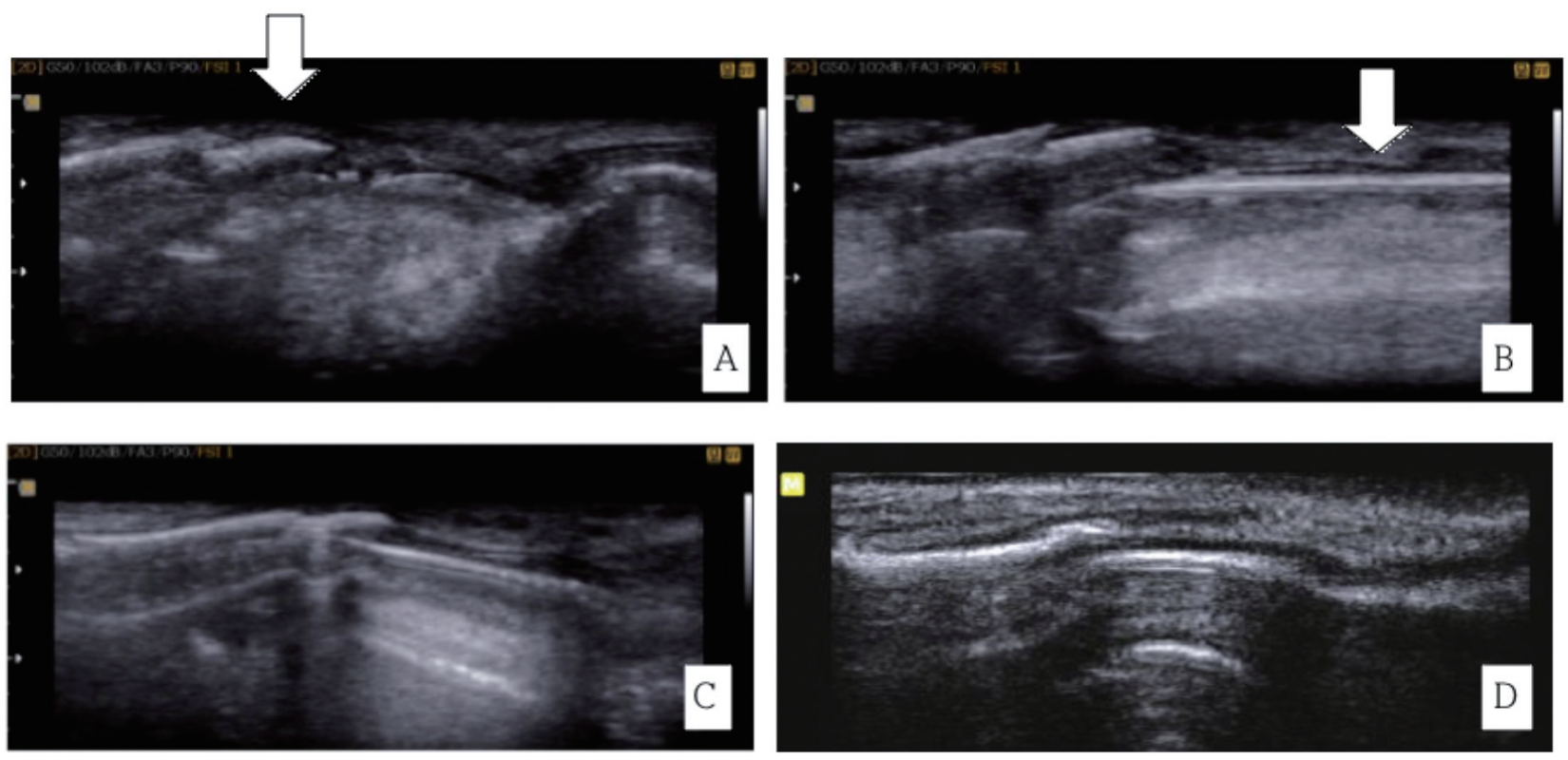

Fig. 1. Ultrasound-images obtained during a closed reduction. (A) Fractured nasal bone (white arrow)-before surgery. (B) Insertion of the fracture reduction apparatus (white arrow). (C) Reduction in progress. (D) Reduced nasal bone fracture. 
was evaluated for nasal bone deviation, bony hump, and for other types of displacement. Each of these criteria was evaluated between 1 ("poor") to 4 ("excellent"), and the mean score between the two rater was used for analysis. The interrater and intrarater reliabilities were assessed using intraclass coefficient. Intra-class coefficients between 0.60 and 0.80 were considered substantial; coefficients greater than 0.80 were excellent [6].

In addition, patient dissatisfaction was evaluated using a questionnaire survey, which focused on aesthetic and functional aspects at 3 months after the reduction operation. For each survey, a

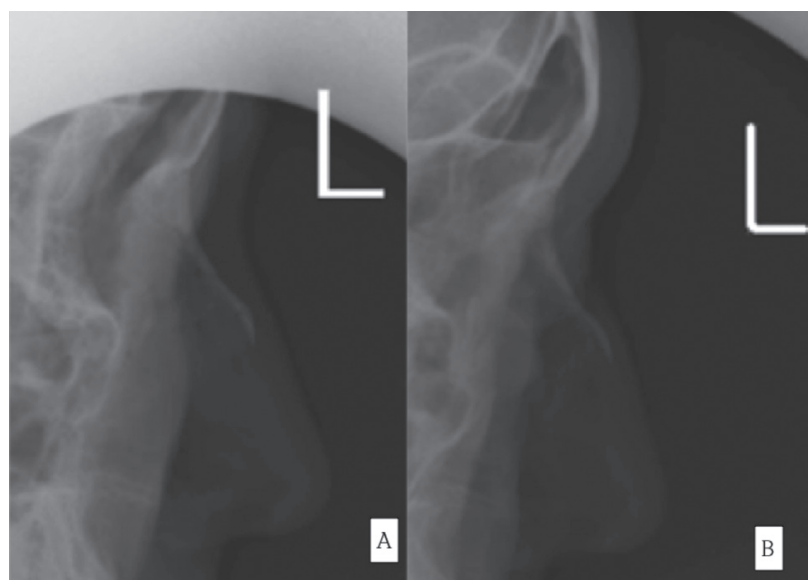

Fig. 3. Preoperative and postoperative X-ray images in a patient who underwent blind closed reduction. (A) Nasal bone fracture is observed. (B) The fracture remains undercorrected at 3 months.

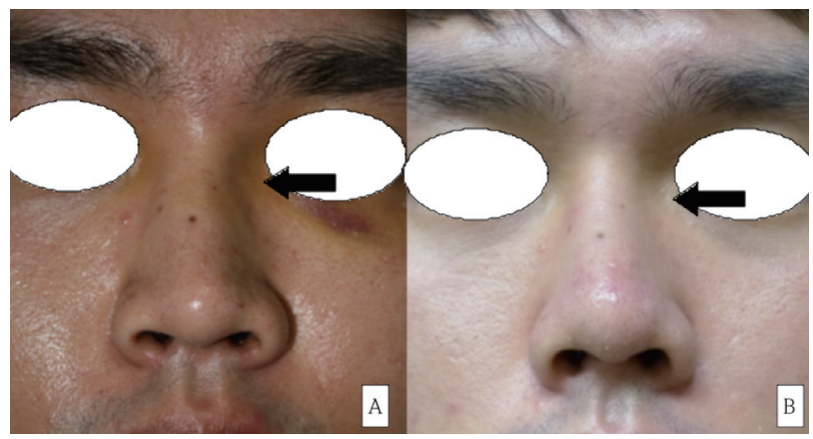

Fig. 4. This 27-year-old male patient presented with a Plane II nasal bone fracture (A) and underwent ultrasound-assisted closed reduction. Follow-up photograph at 3 months (B) after reduction shows correction of indentation (arrows). (A) Indentation caused by the plane II nasal bone fracture marked with an arrow is observed in the 27-year-old male patient. (B) It can be seen with naked eyes that the indentation caused by the nasal bone fracture (marked with an arrow) has been corrected 3 months after the ultrasound-guided closed reduction had been performed. patient answered questions to five aesthetic items and five functional items, all of which referred to post-nasal reduction complications commonly described in the literature [7]. The aesthetic items included complaints regarding the nose shape and consequential stress, the disruption of daily life and interpersonal relationship, and the feeling of depression. The functional items included swelling, nasal congestion, dyspnea, insomnia, and exertional dyspnea. For each time, patients assigned a score between 0 (very satisfied) to 4 (very dissatisfied) (Table 1).

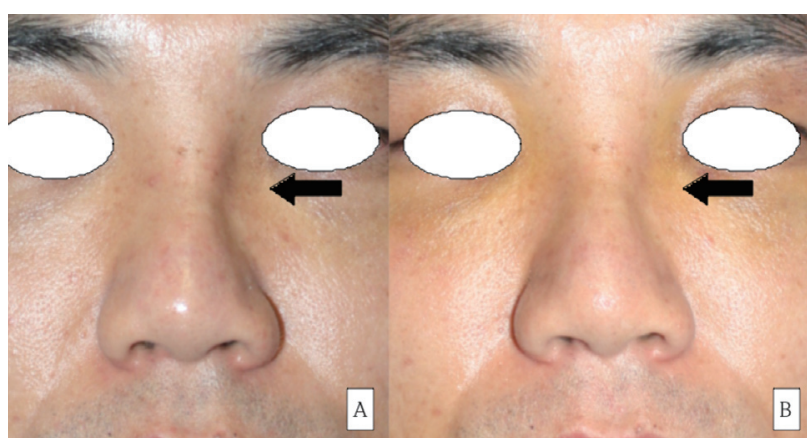

Fig. 5. This 36-year-old male patient also presented with Plane II fracture-with similar indentation to the patient in Fig. 4. However, this patient underwent blind reduction. Unfortunately, the blind reduction resulted in suboptimal correction of the bridge at 3-month visit (arrows).

Table 1. Patient questionnaire after nasal fracture reduction

Aesthetic aspects
1. Are you bothered by the nasal shape?
0 (Not at all), 1 (No), 2 (Not sure), 3 (Yes), 4 (Definitely)
2. Are you under stress because of the problem mentioned above?
0 (Not at all), 1 (No), 2 (Not sure), 3 (Yes), 4 (Definitely)
3. Does this stress disrupt your daily life including work?
O (Not at all), 1 (No), 2 (Not sure), 3 (Yes), 4 (Definitely)
4. Does this stress interfere with your interpersonal relationships?
0 (Not at all), 1 (No), 2 (Not sure), 3 (Yes), 4 (Definitely)
5. Do you feel depressed because of the abovementioned stress?
0 (Not at all), 1 (No), 2 (Not sure), 3 (Yes), 4 (Definitely)
Functional aspects
6. Do you have difficulties in smelling?
0 (Not at all), 1 (No), 2 (Not sure), 3 (Yes), 4 (Definitely)
7. Do you feel that your nose is blocked?
0 (Not at all), 1 (No), 2 (Not sure), 3 (Yes), 4 (Definitely)
8. Do you have difficulties in breathing?
0 (Not at all), 1 (No), 2 (Not sure), 3 (Yes), 4 (Definitely)
9. Do these symptoms interfere with your sleep?
0 (Not at all), 1 (No), 2 (Not sure), 3 (Yes), 4 (Definitely)
10. Do you feel uncomfortable or have difficulty breathing through your
nose during exercise?
0 (Not at all), 1 (No), 2 (Not sure), 3 (Yes), 4 (Definitely)


Within each study group (control and experimental), the results were analyzed for Plane I and II in the Stranc classification [4]. In order to verify the statistical significance, $p$-values were obtained with a 95\% confidence interval by Mann-Whitney test using IBM SPSS ver. 19 (IBM Inc., Armonk, NY, USA).

\section{RESULTS}

The experimental group consisted of 4 patients with Plane I fracture and 10 patients with Plane II fracture. The control group consisted of 3 patients with Plane I fracture and 11 patients with Plane II fracture.

In patients with Plane I fractures, the postoperative outcome scores were 3.6 points for ultrasound-assisted reduction and 3.5 points for blind reduction. In patients with Plane II fractures, the postoperative outcome scores were 3.3 points for ultrasound-assisted reduction and 2.5 points for blind reduction. The intrarater and interrater reliabilities were 96.1\% (95\% confidence interval, 0.916 to 0.982 ) and $93.8 \%$ (0.867 to 0.971$)$, respectively (Table 2 ).

On the questionnaire survey, patients who had Plane I nasal fractures reported a combined score of 6.0 points in the control group and 5.5 points in the experimental group. Patients with Plane II fractures reported a combined score of 9.1 points in the control group and 6.9 points in the experimental group (Table 3).

Specific to Plane II fracture subgroups, patients indicated the highest amount of dissatisfaction, aesthetically, for nasal shape (1.6 points for the control group and 1.5 points for the experimental group), whereas disruption of work was cause for the least amount of dissatisfaction ( 0.7 for the control group and 0.4 for the experimental group). As far as functional outcome was concerned, patients expressed highest amount of dissatisfaction for breathing difficulty during exercise (1.3 for the experimental group and 1.5 for the control group) (Table 4).

Statistical analyses did not discover any significant difference between blind reduction and ultrasound-assisted reduction for patients with minor fractures (Plane I). However, ultrasound-assisted reduction was associated with higher levels of satisfaction among those patients with moderate degrees of nasal fracture (Plane II).

Table 2. Outcomes score were given by two independent, treatmentblinded surgeons who reviewed the X-ray and clinical photographs (preoperative vs. 3 months)

\begin{tabular}{cccc}
$\begin{array}{c}\text { Degree of } \\
\text { fracture }\end{array}$ & $\begin{array}{c}\text { Ultrasound-guided } \\
\text { reduction group }\end{array}$ & $\begin{array}{c}\text { Conventional blinded } \\
\text { reduction group }\end{array}$ & $p$-value \\
\hline Plane I & $3.6(n=4)$ & $3.5(n=3)$ & 0.755 \\
Plane II & $3.3(n=10)$ & $2.5(n=11)$ & 0.007 \\
\hline
\end{tabular}

Table 3. Patient questionnaire scores for each group

\begin{tabular}{lccc}
$\begin{array}{c}\text { Degree of } \\
\text { fracture }\end{array}$ & $\begin{array}{c}\text { Ultrasound-guided } \\
\text { reduction group }\end{array}$ & $\begin{array}{c}\text { Conventional blinded } \\
\text { reduction group }\end{array}$ & $p$-value \\
\hline Plane I & $5.5(n=4)$ & $6.0(n=3)$ & 0.578 \\
Plane II & $6.9(n=10)$ & $9.1(n=11)$ & 0.043 \\
\hline
\end{tabular}

These scores represent the mean of combined scores for each questionnaire completed.

Table 4. The mean scores per questionnaire items among patients with Plane II fractures

\begin{tabular}{|c|c|c|}
\hline Questionnaire & $\begin{array}{l}\text { Ultrasound-guided } \\
\text { reduction group }\end{array}$ & $\begin{array}{l}\text { Conventional blinded } \\
\text { reduction group }\end{array}$ \\
\hline \multicolumn{3}{|l|}{ Aesthetic aspects } \\
\hline 1. Are you bothered by the nasal shape? & 1.5 & 1.6 \\
\hline 2. Are you under stress because of the problem mentioned above? & 0.7 & 1.5 \\
\hline 3. Does this stress disrupt your daily life including work? & 0.4 & 0.7 \\
\hline 4. Does this stress interfere with your interpersonal relationships? & 0.6 & 0.7 \\
\hline 5. Do you feel depressed because of the abovementioned stress? & 0.5 & 0.5 \\
\hline \multicolumn{3}{|l|}{ Functional aspects } \\
\hline 6. Do you have difficulties in smelling? & 0.3 & 0.8 \\
\hline 7. Do you feel that your nose is blocked? & 0.6 & 0.6 \\
\hline 8. Do you have difficulties in breathing? & 0.7 & 0.7 \\
\hline 9. Do these symptoms interfere with your sleep? & 0.3 & 0.5 \\
\hline 10. Do you feel uncomfortable or have difficulty breathing through your nose during exercise? & 1.3 & 1.5 \\
\hline
\end{tabular}




\section{DISCUSSION}

Nasal bone fractures are the most common among facial fractures, and reduction of these fractures are delayed until the edema has subsided. Because of this, nasal fractures are usually addressed at about 7 days after the injury [7]. Fractures can be reduced via closed or open approaches, but most fractures are reduced in closed fashion because of the advantages over open reduction such as simplicity of operation, low risk of infection, and lack of postoperative scars.

The disadvantage of closed reduction is the difficulty in accurately reducing the nasal bones as it is based on the surgeon's sense of touch. Because direct visualization is not possible, closed reduction poses the risk of undercorrection or overcorrection of the fracture. This difficulty can result in increased number of reduction attempts, which is associated with the risk of edema, hemorrhage, inflammation, and nasal synechiae [4]. Particularly in severe fractures (i.e., Plane II or III fractures), soft-tissue edema is significant enough to obscure palpation of the fractured segment. In addition, complex fracture lines it make accurate reduction that much more difficult when compared with minor fractures. If for such reason, reduction is attempted several more times, soft tissues in the vicinity of fractured site may be damaged, which increases the chance of complications such as edema, epistaxis, or inflammation. Chen et al. [3] suggested that closed reduction of traumatic nasal bone fracture was $14 \%-62 \%$ and that $9 \%$ of such cases needed reoperation.

Han et al. [4] noted that the most appropriate time to judge the adequacy of reduction for facial bone fractures is during the operation, while it is still possible to adjust the degree of reduction. It is possible to view the fractured site indirectly, at the time of operation, using portable X-ray, C-arm fluoroscopy, or ultrasonography. Portable X-ray requires re-positioning of the patient to obtain the desired perspective. C-arm fluoroscopy carries the risks of irradiation, and its accuracy was found to be lower than that of ultrasound in evaluating the nasal fracture $[5,8]$.

Ultrasound is a favorable imaging modality to evaluate the fracture site during closed reduction of a nasal fracture because of the thinness of nasal skin and mucosal layer under the skin [8]. In a previous study, Abu-Samra et al. [9]. had compared the usefulness of ultrasound in closed reduction of nasal fractures. The authors compared evaluation of the nasal fracture between ultrasound and plain radiography and found that ultrasound was superior in diagnosing the nasal bone fracture, as the ultrasoundguided method was $100 \%$ sensitive while plain radiography was only $59 \%$ sensitive. However, the patient-reported satisfaction revealed no significant outcomes difference between those treated with ultrasound-assisted closed reduction and those treated with conventional closed reduction. The authors argued that the lack of significant difference was most likely due to the fact that patients who underwent the reduction of a nasal bone fracture as reconstruction had lower aesthetic demands than those who underwent surgery for cosmetic purpose [9]. However, their study had not distinguished this comparison according to the extent of fracture.

In the present study, we investigated outcomes following ultrasound-guided closed reduction to overcome the limitations of blind approach. In those patients with Plane I fractures, patientsurvey scores were not statistically different between the groups, as the average of combined scores were 5.5 points with ultrasound and 6.0 points without ultrasound ( $p=0.578$ ). Among patients with Plane II fractures, however, patients who underwent blind reduction reported a significantly higher level of dissatisfaction (9.1 points) when compared to that of the ultrasound-assisted closed reduction (6.9 points) $(p=0.043)$.

Potential reasons for ultrasound-guided closed reductions increasing patient satisfaction for Plane II fractures are as follows. Fracture patterns are complex and edema is severe in Plane II fractures, which makes it difficult to determine the exact fracture patterns solely with palpation. Ultrasound imaing is helpful in such cases as it allows for a more accurate reduction while decreasing the number of attempts at reduction, which can minimize the damage to soft tissues including the mucous membrane. In addition, ultrasound allows for intraoperative identification of undercorrection and overcorrection of fracture displacement. In this context, our study result suggests that ultrasound-guided closed reduction is superior to blind closed reduction in those patients with Plane II nasal fractures. Future studies should focus on this subset group of nasal fracture patients. 


\section{REFERENCES}

1. Hwang SM, Kim JH, Kim HD, Jung YH, Kim HI. C-arm fluoroscopy for accurate reduction of facial bone fracture. Arch Craniofac Surg 2013;14:96-101.

2. Lim KW, Kim JC, Kim SH, Kim SH. A clinical and statistical analysis of the facial bone fractures: from 1994 to 1998. J Korean Soc Plast Reconstr Surg 1999;26:1151-9.

3. Chen RF, Chen CT, Hao Chen C, Liao HT, Chen YR. Optimizing closed reduction of nasal and zygomatic arch fractures with a mobile fluoroscan. Plast Reconstr Surg 2010;126:554-63.

4. Han DS, Han YS, Park JH. A new approach to the treatment of nasal bone fracture: the clinical usefulness of closed reduction using a Carm. J Plast Reconstr Aesthet Surg 2011;64:937-43.

5. Imai T, Michizawa M, Yamamoto N, Kai T. Closed reduction of man- dibular condyle fractures using C-arm fluoroscopy: a technical note. Oral Surg Oral Med Oral Pathol Oral Radiol 2013;115:e4-9.

6. Yu MS, Kim BH, Kang SH, Lim DJ. Combined use of crushed cartilage and fibrin sealant for radix augmentation in Asian rhinoplasty. Plast Reconstr Surg 2015;135:293e-300e.

7. Lim KR, Kim HI, Ahn SM, Hwang SM, Jung YH, Song JK. Clinical analysis of the nasal bone fracture. J Korean Cleft Palate-Craniofac Assoc 2011;12:81-5.

8. Gurkov R, Clevert D, Krause E. Sonography versus plain x rays in diagnosis of nasal fractures. Am J Rhinol 2008;22:613-6.

9. Abu-Samra M, Selmi G, Mansy H, Agha M. Role of intra-operative ultrasound-guided reduction of nasal bone fracture in patient satisfaction and patient nasal profile (a randomized clinical trial). Eur Arch Otorhinolaryngol 2011;268:541-6. 